\title{
Can assimilation of satellite observations improve subsurface biological properties in a numerical model? A case study for the Gulf of Mexico
}

\author{
Bin Wang ${ }^{1}$, Katja Fennel ${ }^{1}$, Liuqian $\mathrm{Yu}^{1,2}$ \\ ${ }^{1}$ Department of Oceanography, Dalhousie University, Halifax, Nova Scotia, Canada \\ ${ }^{2}$ Department of Ocean Science, The Hong Kong University of Science and Technology, \\ Kowloon, Hong Kong
}

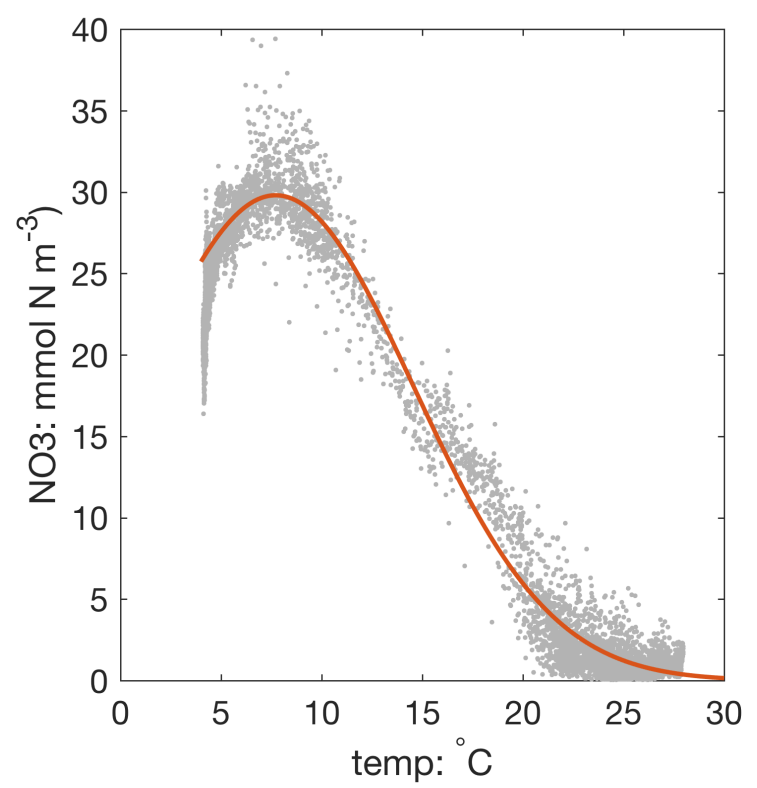

Figure S1. Empirical relations of temperature-NO3 derived from World Ocean Atlas in the Gulf of Mexico 



Figure S2. Vertical profiles of chlorophyll (a), phytoplankton (b), and POC (c) from BOEM floats and model experiments.

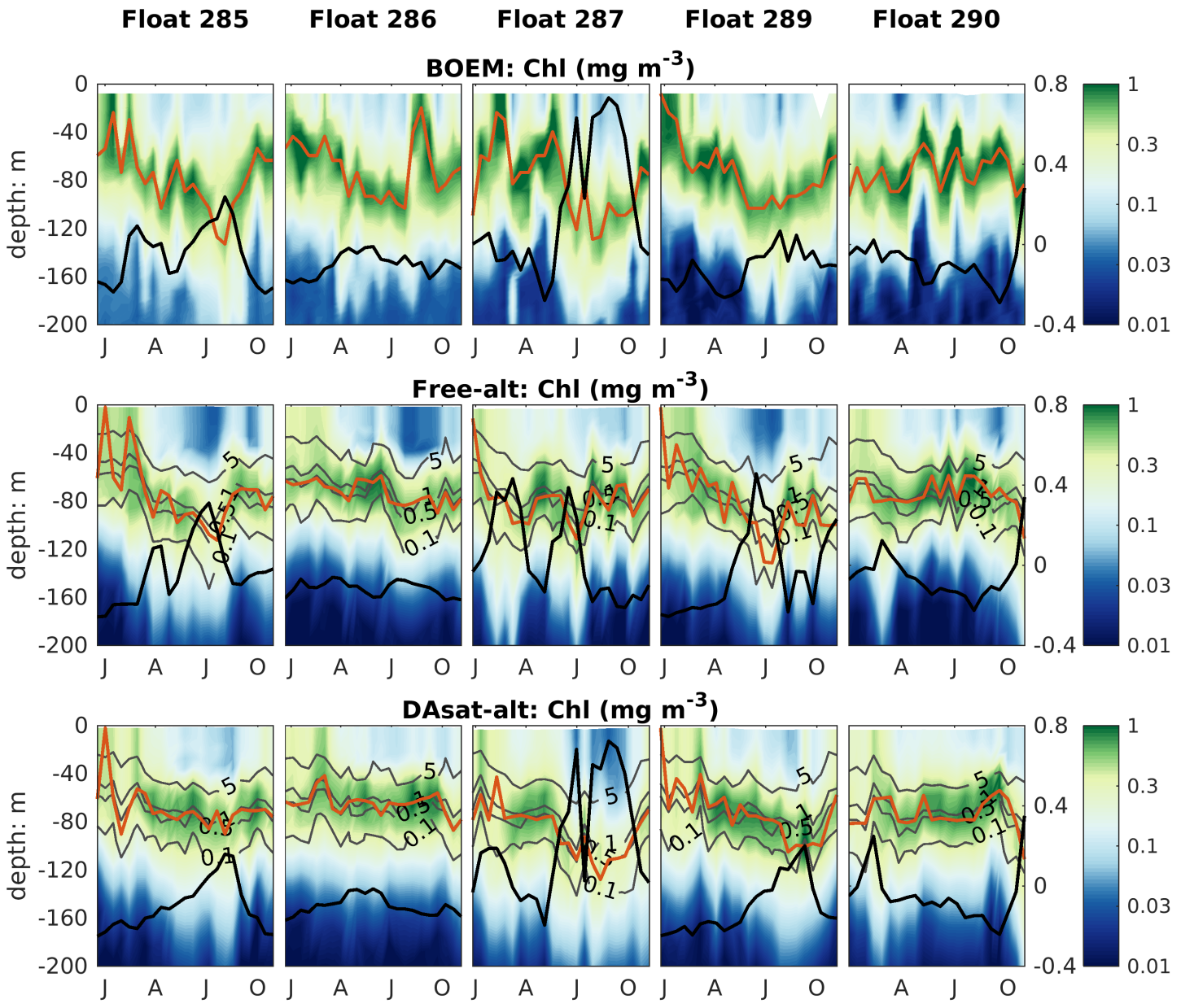

Figure S3. Vertical distributions of chlorophyll from BOEM floats, Free-alt run, and DAsat-alt run. 

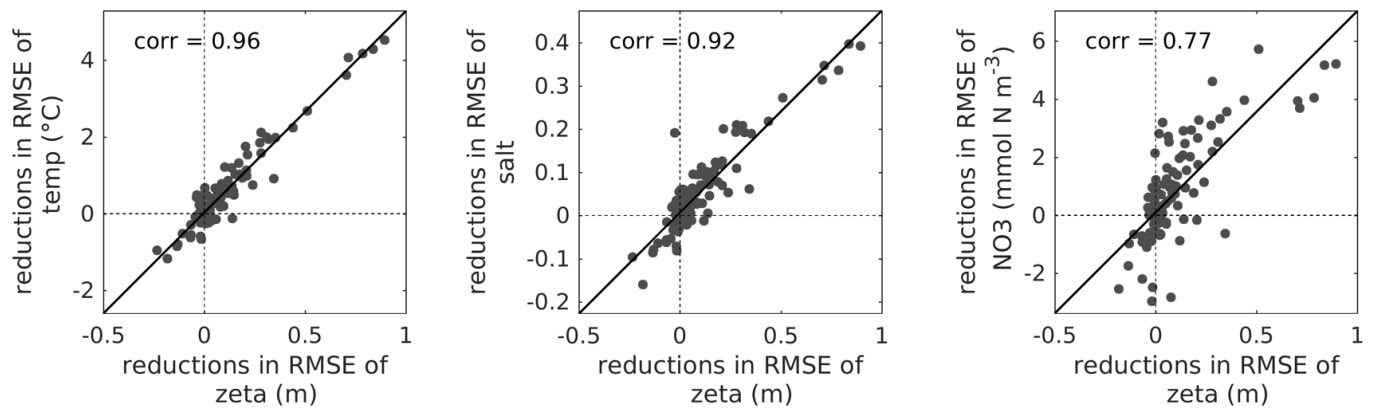

Figure S4. Correlations of improvement between zeta and temperature, salinity, and NO3 